
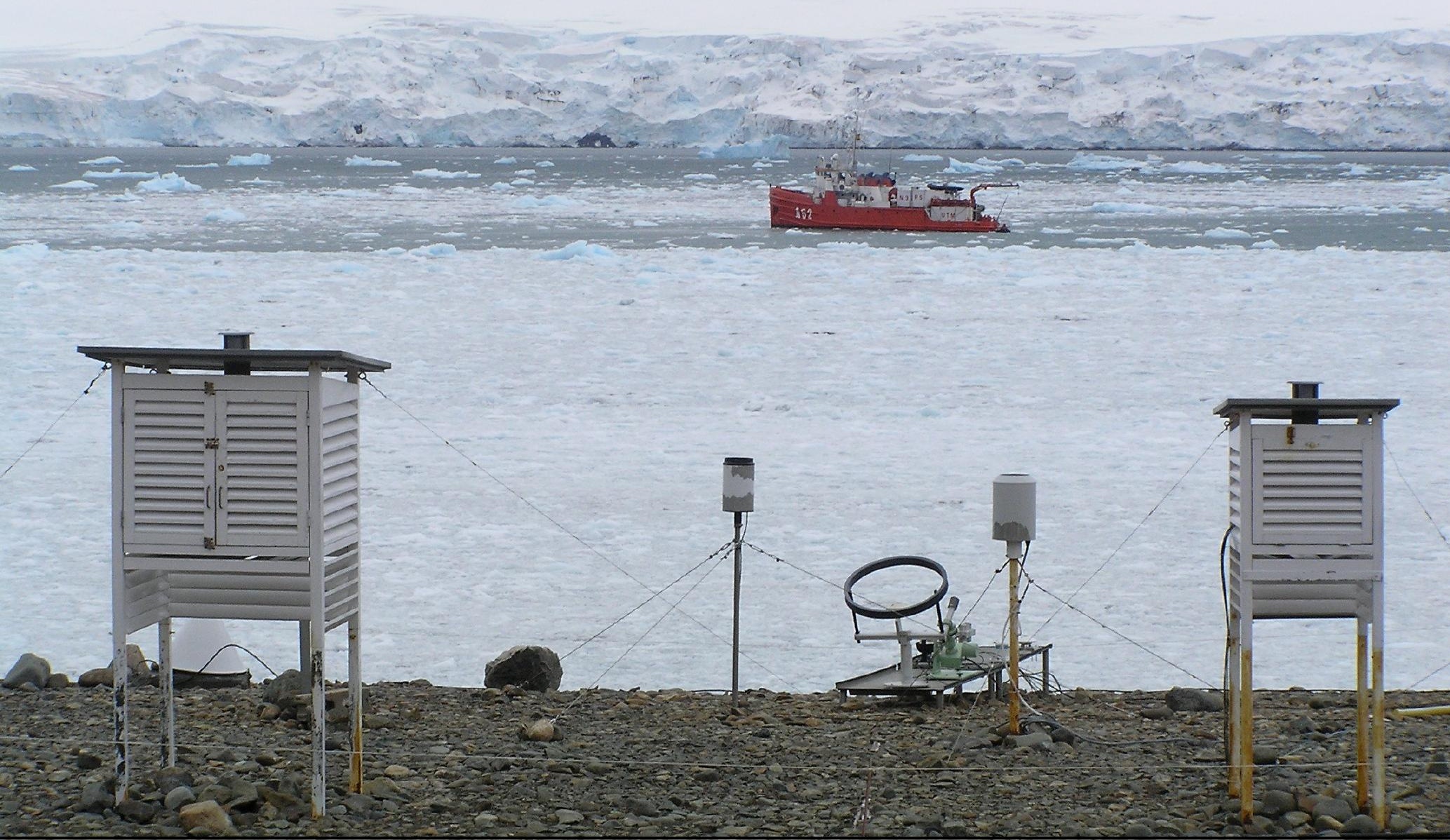

\title{
Climatología y observaciones
}

DOI: $10.31978 / 014-18-009-X .09$

Andrés Chazarra Bernabé, CÉsar Rodríguez Ballesteros y Celia Milagros Flores Herráez Área de Climatología y Aplicaciones Operativas, Agencia Estatal de Meteorología (AEMET)

La palabra Clima, etimológicamente, significa inclinación, y se refiere a la oblicuidad de los rayos solares que llegan al suelo, la cual varía según las horas del día, la época del año y la latitud del lugar.

\section{Curso de climatología - J. M. JANSÀ GUARDIOLA}

Un ingrediente esencial para la elaboración de predicciones acertadas es un conocimiento preciso y lo más exacto posible del estado actual de la atmósfera. Los sistemas de observación son, por tanto, esenciales para los sistemas de predicción. La predecibilidad, que tiene que ver con la capacidad para predecir el tiempo correspondiente a cada día y lugar, depende de las situaciones o configuraciones atmosféricas y, así mismo, el contraste de estas configuraciones con la climatología aumenta nuestro conocimiento de esa predecibilidad y puede ayudar a mejorar su formulación. De este modo, la predecibilidad está relacionada en cierta medida con la geografía y con la climatología correspondientes al fenómeno y al lugar donde realizamos la predicción. Climatología y predecibilidad guardan así una relación a la que se debe prestar atención. Nos acercamos en este capítulo a la climatología y a algunas de las redes de observación utilizadas en AEMET con fines climatológicos.

Palabras clave: climatología, observación de la atmósfera, redes de observación, normales climatológicas, Banco Nacional de Datos Climatológicos, control de calidad y almacenamiento de datos climatológicos.

Imagen parte superior: jardín meteorológico de AEMET en la Antártida en su antigua ubicación, en segundo plano el buque Las Palmas en medio del brash, 8 de enero de 2007, Base Antártica Española Juan Carlos I, isla Livingston, South Shetland, Antártida. El jardín se trasladó posteriormente durante la campaña 2008-2009 con motivo de las obras de la nueva base. Fotografía tomada por FRANCISCO VASALLO LÓPEZ. 


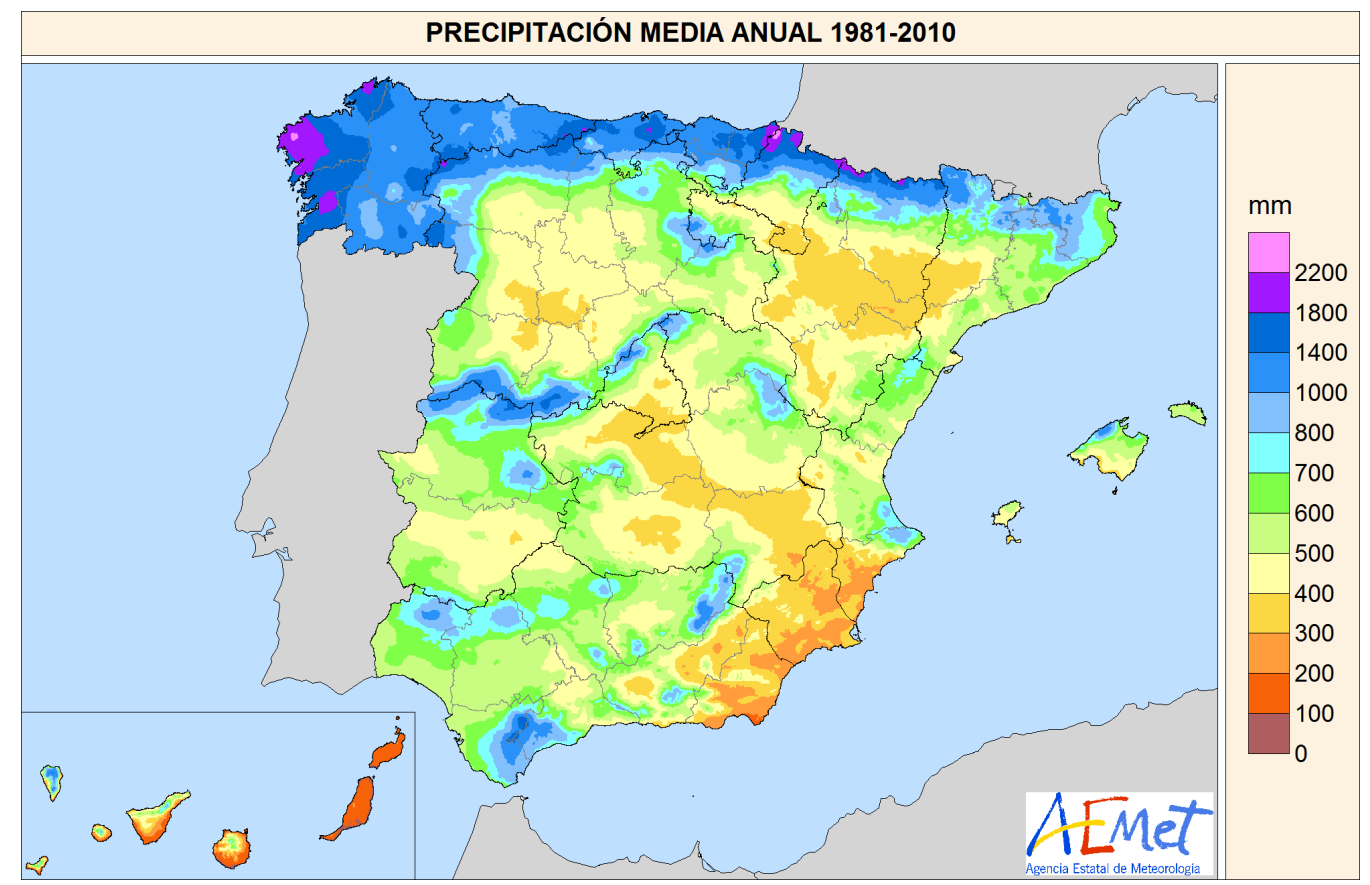

Figura 9.1: Mapa de la precipitación media anual en España correspondiente al período 1981-2010. Fuente: Agencia Estatal de Meteorología (AEMET).

\subsection{Climatología y clima}

Según la definición de la Guía de Prácticas Climatológicas de la Organización Meteorológica Mundial [5], la climatología consiste en el estudio del clima, sus variaciones y extremos y su influencia en varias actividades, sobre todo (aunque no exclusivamente) en los ámbitos de la salud, la seguridad y el bienestar humanos.

A su vez, se entiende por clima las condiciones meteorológicas normales correspondientes a un lugary a un período de tiempo determinados.

Conviene tener presente la diferencia entre tiempo y clima: el tiempo es el estado atmosférico en un momento y lugar concretos, tiene por tanto un carácter instantáneo, mientras que el clima hace referencia al estado medio del tiempo, así como sus variaciones y extremos, en un lugar y un periodo de tiempo determinados. Es decir, el clima es una síntesis de los diferentes tiempos o estados atmosféricos que podemos esperar que ocurran en cada lugar y momento. Así, cuando calificamos el clima actual del sureste de la península ibérica como seco, nos estamos refiriendo a que las precipitaciones son generalmente escasas en comparación con otras regiones, pero evidentemente podrá haber días en los que el tiempo sea lluvioso y se registren precipitaciones abundantes e intensas.

Para describir el clima de un lugar se utilizan descrip- ciones estadísticas de los valores que toman los principales elementos climáticos como la temperatura del aire, la precipitación, la humedad, el viento, la insolación, etc., las cuales permiten caracterizar tanto el estado medio del tiempo como su variabilidad y sus tendencias en un periodo de tiempo determinado. Así, el clima de un lugar queda caracterizado mediante valores medios, máximos, mínimos, distribuciones de probabilidad y otros estadísticos de magnitudes medibles relacionadas con los elementos climáticos (ejemplos en Figura 9.1 y en el Atlas Climático Ibérico [1] http: //www . aemet.es/documentos/es/conocermas/ recursos_en_linea/publicaciones_y_estudios/ publicaciones/Atlas-climatologico/Atlas. pdf).

Hasta bien entrado el siglo XX se consideraba que las variaciones del clima eran generalmente lentas y graduales, siendo frecuentes en los textos de climatología de la época las referencias al clima de un lugar como una característica apenas invariante en el tiempo. Sin embargo, el cambio climático observado en los últimos decenios del siglo XX, el cual ha dado lugar a una notable variación estadística tanto del estado habitual del clima como de su variabilidad a escala global, ha supuesto un reto para los climatólogos. A partir de entonces, se ha comenzado a considerar el clima y sus variaciones como una consecuencia de las interacciones entre los componentes del sistema climático, el cual está formado por la atmósfera, la superficie 
terrestre, la nieve y el hielo, los océanos y otras masas de agua y los organismos vivos. El sistema climático presenta una variabilidad interna debida a las interacciones entre los componentes del sistema climático, las cuales ocurren a todas las escalas espaciales y temporales y, por otro lado, una variabilidad externa que depende de factores externos al sistema, tanto naturales (variaciones solares, erupciones volcánicas, etc.) como antropogénicos (alteraciones de la composición de la atmósfera y de la superficie terrestre).

\subsection{Caracterización del clima a partir de datos climatológicos}

La caracterización del clima se realiza a partir de los datos recogidos en las redes de estaciones climáticas. La abundante información almacenada en las bases de datos climáticos es analizada y resumida en un número relativamente pequeño de valores que se obtienen al aplicar métodos estadísticos. Si se escogen de forma adecuada, estos valores estadísticos permiten resaltar y poner de manifiesto las principales características del clima de forma sencilla y fácilmente interpretable.

Son muchos los estadísticos que pueden calcularse a partir de los conjuntos de datos y que resultan de utilidad para la descripción del clima. Los más habitualmente empleados en climatología comprenden: medidas de tendencia central, como la media y la mediana, las cuales indican un valor central alrededor del cual tienden agruparse los datos de una variable climática; medidas de variabilidad, como la desviación típica y el rango de los datos (diferencia entre el valor máximo y el mínimo); distribuciones de probabilidad, percentiles, valores extremos, etc.
También es habitual el empleo de índices climáticos, los cuales sintetizan en un único valor numérico relaciones más o menos complejas entre elementos climáticos que conservan cierto sentido físico y que pueden utilizarse para el seguimiento de un proceso determinado, como por ejemplo el índice de PALMER para la gravedad de la sequía o los índices utilizados en las clasificaciones climáticas (OMM, Guía de Prácticas Climatológicas 2011 [5]). Además, el estudio de las series temporales, formadas por las observaciones ordenadas según la secuencia en la que se efectuaron, permite determinar de forma cualitativa y cuantitativa las variaciones del clima en el tiempo [3].

A la hora de presentar los resultados, en climatología se utilizan con frecuencia tablas, series de datos, mapas climáticos y gráficos con el objeto de mostrar y sintetizar de la manera más clara e intuitiva posible la información contenida en los conjuntos de datos y en los estadísticos derivados de ellos.

Para que los valores estadísticos de las observaciones realizadas en observatorios distintos sean comparables debe tenerse especial cuidado en que las medidas se hayan realizado mediante procedimientos análogos, empleando instrumentos y métodos de medida compatibles entre sí y, además, que el periodo de tiempo a lo largo del cual se han realizado las medidas sea el mismo ya que, como hemos visto, el clima y, por tanto, los valores que toman los elementos climáticos en cada lugar pueden variar de forma significativa a lo largo del tiempo. Por ello, tiene especial importancia en climatología el concepto de normal climatológica que vemos a continuación.

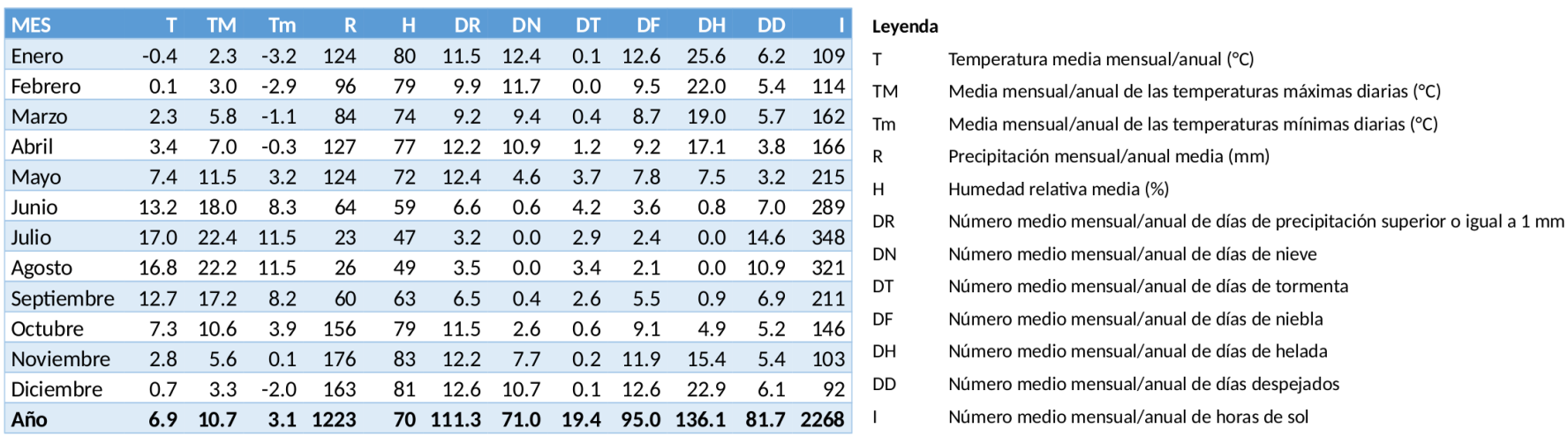

Figura 9.2: Valores climatológicos normales del período 1981-2010 del observatorio del Puerto de Navacerrada. Fuente: AEMET. 


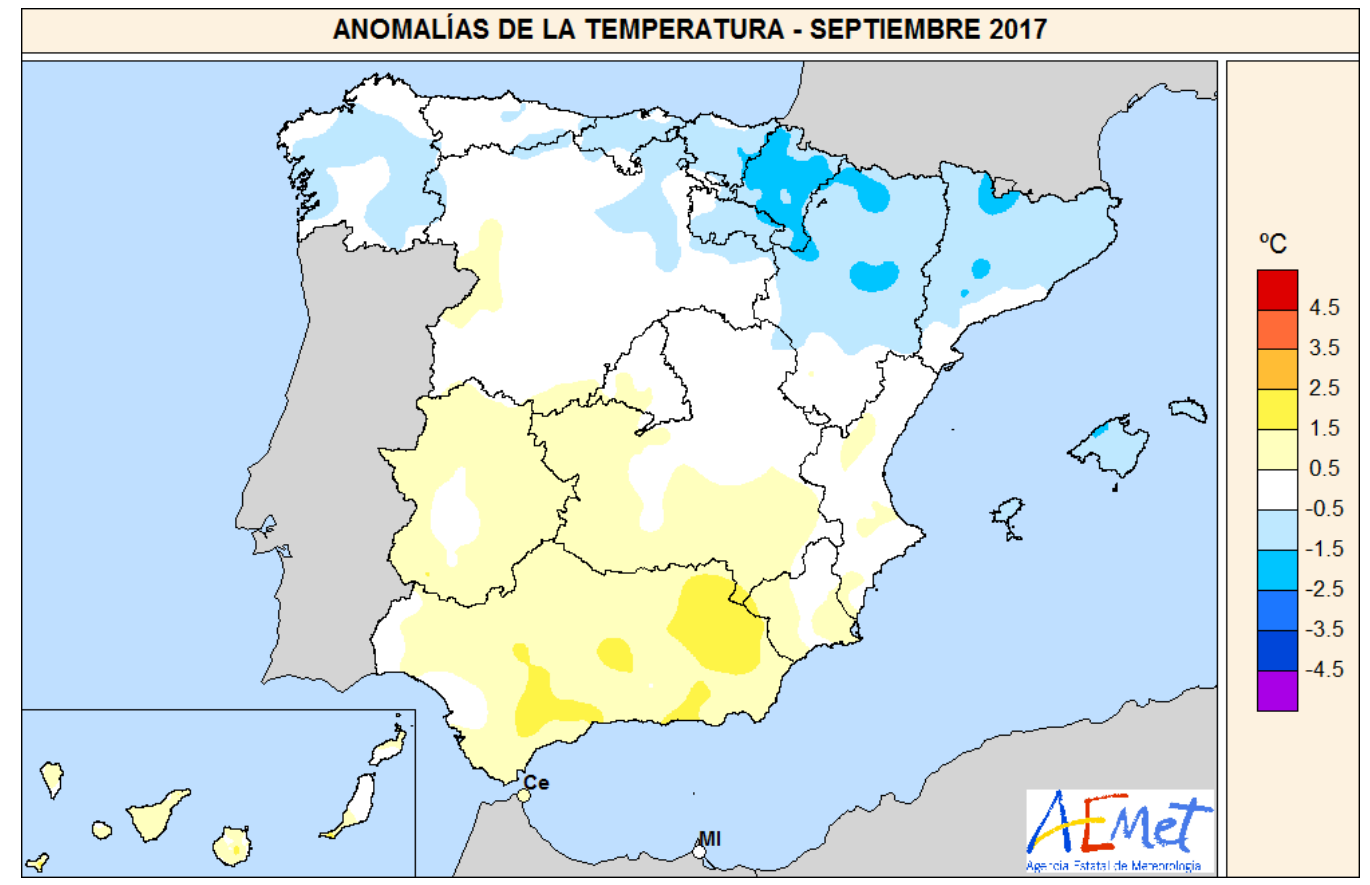

Figura 9.3: Mapa de anomalías de la temperatura media de septiembre de 2017 respecto de los valores normales del periodo 1981-2010. Uno de los fines principales de los valores normales es servir de referencia para evaluar las condiciones climáticas observadas en una fecha o un período de tiempo determinados. Fuente: AEMET.

\subsection{Normales climatológicas}

Las normales climatológicas son las medias de los datos climatológicos calculadas para un periodo uniforme y relativamente largo que comprende al menos tres periodos consecutivos de diez años (ejemplo en Figura 9.2 en la página anterior). Según la Organización Meteorológica Mundial, el uso de normales tiene dos finalidades principales:

- Constituyen una referencia para evaluar las condiciones climáticas actuales o recientes, permitiendo obtener, por ejemplo, anomalías térmicas (diferencias de la temperatura observada en un lugar determinado respecto de la normal en ese lugar) que pueden compararse con las calculadas en otro lugar distinto, siempre que el periodo de referencia sea el mismo (ver Figura 9.3).

- Se utilizan además con fines de predicción, como indicadores de las condiciones que es probable que se observen en un determinado lugar en el futuro.

Las normales climatológicas reglamentarias o estándares se establecieron por la Organización Meteorológica Mundial a comienzos del siglo XX con el objeto de que los servicios meteorológicos de los distintos países utilizaran los mismos periodos de referencia, de forma que los valores normales y las anomalías fueran comparables en todo el mundo. Inicialmente, se escogieron periodos consecutivos de 30 años, comenzando en el primer día del siglo XX: 1 de enero de 1901 a 31 de diciembre de 1930, 1 de enero de 1931 a 31 de diciembre de 1960, 1 de enero de 1961 a 31 de diciembre de 1990 , etc.

Hasta bien entrado el siglo pasado se consideraba que el clima es fundamentalmente constante para escalas temporales de décadas a siglos y que las variaciones observadas respecto de ese estado medio se debían principalmente al método de muestreo. Por tanto, los valores medios debían converger a ese estado medio al tomar periodos de tiempo suficientemente largos, y se consideró que treinta años eran suficientes para filtrar las fluctuaciones.

Sin embargo, durante la segunda mitad del siglo XX fueron cada vez mayores las evidencias de que el clima es cambiante (IPCC, 2014 [2]), por lo que los valores normales dejan pronto de tener validez como indicadores del clima presente y futuro [4] (ver Figura 9.4 en la página siguiente). Por este motivo, la Organización Meteorológica Mundial modificó recientemente los periodos de referencia para el cálculo de las normales climatológicas reglamentarias, de forma que siguen siendo períodos consecutivos de treinta años, pero pasando a actualizarse cada diez años, abarcando desde un año que termina en 1 hasta un año terminado en 0 . Es decir, ahora las normales reglamentarias incluyen periodos de treinta años que van del 
1 de enero de 1981 al 31 de diciembre de 2010, del 1 de enero de 1991 al 31 de diciembre de 2020, y así sucesivamente. Los organismos responsables deben calcular las normales climatológicas reglamentarias lo más pronto posible una vez finalizado cada decenio y los períodos de las normales climatológicas reglamentarias deben respetarse siempre que sea posible a fin de ofrecer una base uniforme para las comparaciones de valores en todo el mundo.

Una cuestión planteada a menudo a los climatólogos es por qué no se actualizan las normales climatológicas con una frecuencia mayor, por ejemplo, cada año. Si bien es cierto que podría mejorarse ligeramente la capacidad predictiva de los valores normales al utilizarse así siempre el periodo de treinta años más reciente, una actualización frecuente tiene el inconveniente de que es necesario volver a calcular no solo los propios valores normales, sino también todos aquellos conjuntos de datos y productos que utilizan las normales como referencia, resultando un proceso excesivamente costoso en esfuerzo y tiempo. Por otra parte, cuando las normales se utilizan como referencia para la vigilancia y el análisis del clima, una actualización demasiado frecuente de las normales resultaría más un inconveniente que una ventaja ya que, al cambiar continuamente los valores de referencia, no resultarían comparables las anomalías y otros estadísticos calculados en diferentes estudios. Por este motivo, la Organización Meteorológica Mundial ha establecido el 1961-1990 como periodo de referencia fijo para la evaluación a largo plazo del cambio climático y de la variabilidad del clima, de forma que todos los estudios sobre cambio climático que se realicen ofrezcan resultados comparables entre sí. Este período permanecerá en principio fijo para siempre, o al menos hasta que exista un motivo científico convincente para cambiarlo, según la OMM.

\subsection{La importancia de las obser- vaciones climatológicas}

Prácticamente todos los estudios climatológicos utilizan datos procedentes de las redes de observación climatológicas, ya sean medidas directas registradas en un momento determinado o bien valores normales u otros estadísticos obtenidos a partir de series de observaciones. Resulta evidente que la calidad de los resultados que se obtengan dependerá en gran medida de la calidad de los datos originales, siendo por tanto fundamental procurar que las medidas se realicen siguiendo los requerimientos y recomendaciones de la Organización Meteorológica Mundial para que los datos de los distintos observatorios sean comparables entre sí y ofrezcan la calidad necesaria para realizar los estudios climatológicos a partir de ellos.

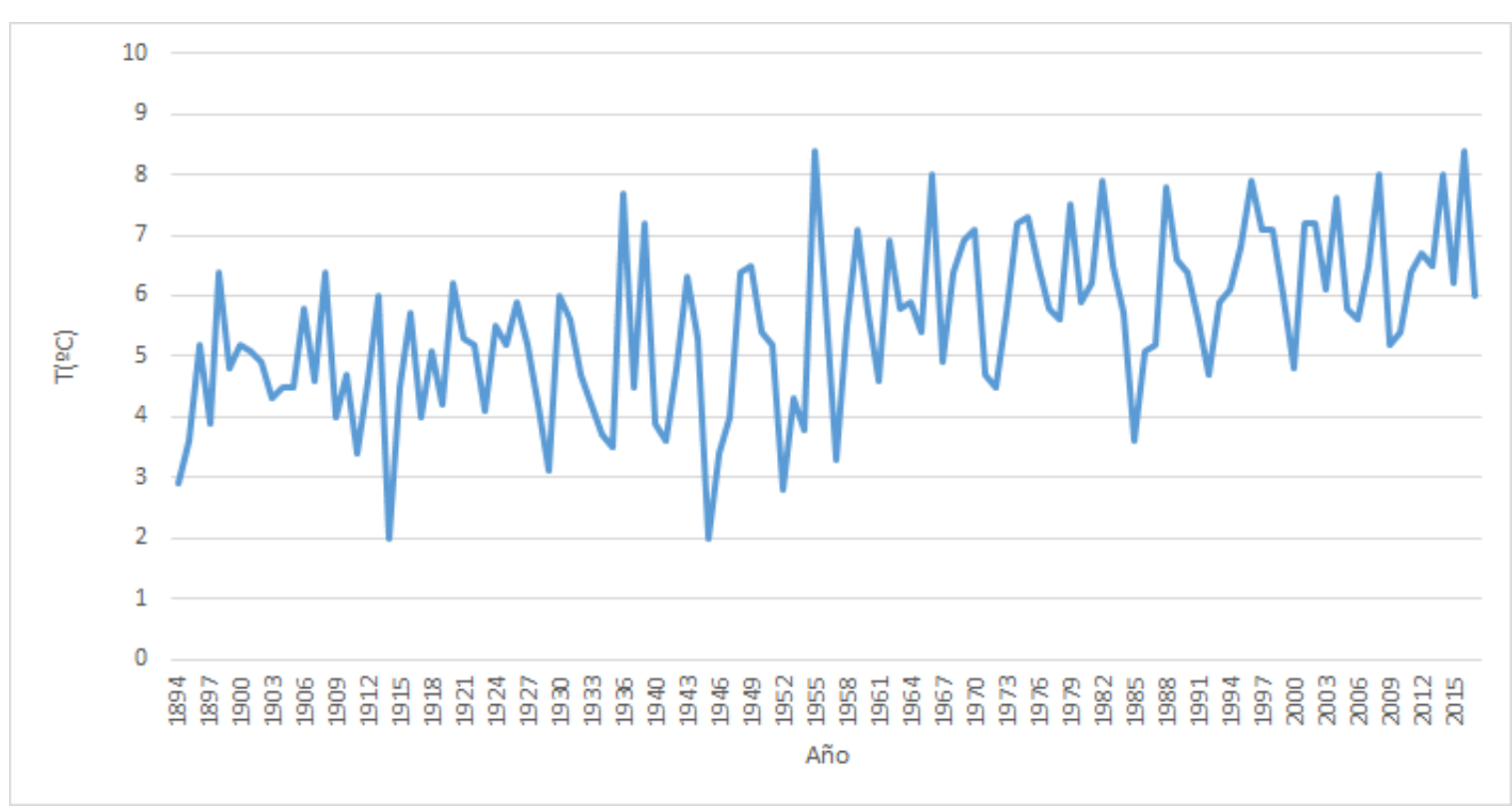

Figura 9.4: Temperatura media mensual de enero en el observatorio de Madrid-Retiro desde 1984. Las series largas de observaciones resultan fundamentales para el estudio de la variabilidad climática. Fuente: AEMET. 
En la medida de lo posible, los datos que se empleen para calcular normales y otros estadísticos climatológicos deben ser homogéneos, prestando especial atención a la posibilidad de que haya habido durante el periodo considerado factores que hayan alterado la homogeneidad de la serie de datos, como pueden ser cambios de emplazamiento, cambios en el entorno, cambios en el procedimiento de observación (por ejemplo, en la hora de observación), cambios en los tipos de instrumentos o en su exposición y cambios en el proceso de datos. La mejor manera de preservar la homogeneidad de un registro es evitar en la medida de lo posible todos estos cambios. Sin embargo, la mayoría de las series climáticas de larga duración se ven afectadas en menor o mayor medida por factores como los anteriormente mencionados que pueden ocasionar heterogeneidades en las series. Existen métodos estadísticos para estudiar la homogeneidad de un conjunto de datos y es posible realizar correcciones en las series de datos para eliminar las inhomogeneidades detectadas, especialmente cuando se dispone de información (metadatos) acerca de las fechas de los cambios y se dispone además de series paralelas suficientemente largas para comparar y corregir los datos antes y después de los cambios. También existen métodos estadísticos que utilizan series de referencia homogéneas para comparar con las series que presentan problemas de homogeneidad y proceder a su corrección.

Así mismo, a menudo existen lagunas en las series de datos que pueden distorsionar de forma significativa los resultados a la hora de calcular estadísticos climatológicos. Si, por ejemplo, calculamos el valor normal de la precipitación anual de una estación en la que falta el valor de un año que fue especialmente lluvioso, el resultado será inferior al que se obtendría si se considerara ese valor. Como es habitual que exista correlación entre los datos climáticos a lo largo del tiempo, la falta consecutiva de observaciones suele tener una mayor repercusión a la hora de calcular estadísticos que la falta del mismo número de observaciones aleatoriamente repartidas durante el mismo período de tiempo. En esos casos es recomendable efectuar, previamente al cálculo de los valores normales y de otros estadísticos, un relleno de las lagunas en el que se estiman los datos faltantes a partir de los valores observados en estaciones cercanas.

\subsection{El Banco Nacional de Datos Climatológicos de AEMET}

El Banco Nacional de Datos Climatológicos de AEMET es una herramienta fundamental en todos los procesos relacionados tanto con la caracterización climática de un lugar como con las labores de Vigilancia del Clima en tiempo cuasi-real o la generación de aplicaciones dirigidas a dar respuesta a las demandas de diferentes sectores de la sociedad. En él se archivan y gestionan principalmente datos meteorológicos procedentes de las diferentes redes de observación de superficie.

AEMET, por su pertenencia a la OMM (Organización Meteorológica Mundial), cumple con los requisitos establecidos por el Sistema Mundial de Observación (SMO) que pertenece al Programa para la Vigilancia Meteorológica Mundial de la OMM [6]. Las observaciones meteorológicas de superficie forman parte del SMO y en ellas se registra información de diferentes variables meteorológicas como son presión, viento, temperatura, humedad relativa, etc. Las distintas redes de observación meteorológica de superficie responden a las diferentes escalas de los fenómenos meteorológicos y a la necesidad de conseguir una buena observación. Por definición, la observación meteorológica consiste en la evaluación o medida de los distintos elementos meteorológicos con el fin de caracterizar el estado físico de la atmósfera en un lugar y momento determinados. Los requisitos referentes a la precisión de los datos, la frecuencia de las observaciones, la distribución espacial de las estaciones así como su densidad, dependen de las diferentes escalas de los fenómenos meteorológicos que han de analizarse. Las escalas horizontales están estrechamente relacionadas con las escalas de tiempo de los fenómenos, por lo que existirán redes de superficie que van desde una escala planetaria $(>3000 \mathrm{~km})$ relacionada con las ondas largas de la troposfera superior hasta la microescala $(<100 \mathrm{~m})$ relacionada con la meteorología agrícola, la evaporación u otros efectos microclimáticos.

Para garantizar la calidad de las observaciones y promover la compatibilidad y estandarización internacional de la instrumentación y de los métodos de observación utilizados, está el Programa de Instrumentos y Métodos de Observación de la OMM que se lleva a cabo a través de la Comisión de Instrumentos y Métodos de Observación (CIMO [6]). 


\section{T2M FROM SYNOP \\ MEAN OBSERVATION [K] (USED) \\ DATA PERIOD $=$ 2017-11-12 $21-2017-11-1409$ \\ $\mathrm{EXP}=0001, \mathrm{CHANNEL}=1$ \\ Min: \\ GRID: $0.50 \times 0.50$}

282.652

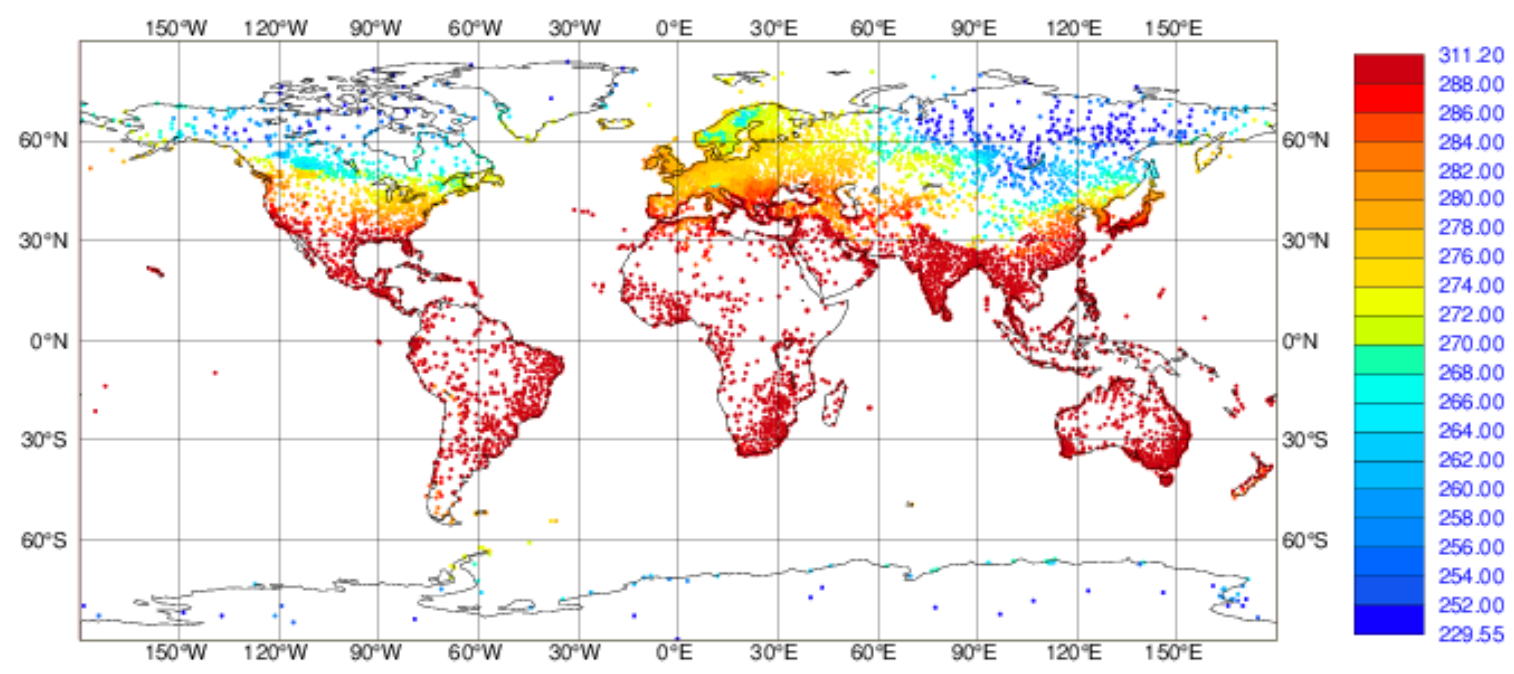

Figura 9.5: Mapa con la temperatura a dos metros del suelo observada en la red de estaciones sinópticas de superficie que se utiliza en el sistema de asimilación de datos del ECMWF. Fuente: ECMWF.

Como resultado de las necesidades planteadas, de los requisitos de intercambio de información mundial tanto meteorológica como climatológica y la necesidad de caracterizar lo mejor posible la climatología de un lugar, surgen las diferentes redes de observación de superficie en AEMET.

Para el intercambio de datos de estaciones meteorológicas de superficie a nivel mundial (Figura 9.5) en tiempo real y asociado a las necesidades de la Región VI de la OMM, España dispone de la Red Sinóptica Básica Regional (RSBR) y varias de dichas estaciones forman parte a su vez de la Red Climatológica Principal.

La RSBR de superficie está formada por un conjunto de estaciones dotadas de personal, con una resolución espacial entre 100 y 150 km, que miden presión, temperatura, viento, humedad, precipitación, tiempo presente, visibilidad, nubosidad, temperatura del suelo y del subsuelo, insolación, estado del suelo y, en el caso de las estaciones costeras, temperatura del agua del mar, oleaje y estado del mar. La frecuencia de transmisión de la información es horaria. El objeto de la red es atender el Programa de la Vigilancia Meteorológica Mundial de la OMM y el programa EUCOS de EUMETNET (http://eumetnet. eu/activities/observations-programme/ current-activities/programme-management/), así como su utilización en modelos numéricos globales y regionales (sec. 10.1 en la página 130). La necesidad de conseguir una adecuada representatividad de las estaciones de la RSBR supone que la ubicación de las mismas, su entorno y la exposición de los instrumentos sean adecuados y esté garantizada su permanencia durante, al menos, 30 años.

La red RSBR se complementa a su vez con la Red Sinóptica Complementaria (RSC). El conjunto de las estaciones que forman parte de la RSC y las de la RSBR, permiten cubrir todo el territorio nacional con una resolución espacial inferior a $80 \mathrm{~km}$, y en conjunto forman la Red Sinóptica Nacional (RSN). Su objetivo es la vigilancia meteorológica y el intercambio de datos con otros países e instituciones, así como su utilización en modelos numéricos de área limitada (sec. 10.1 en la página 130).

No obstante, como los requerimientos de información meteorológica son cada vez mayores, estas dos redes anteriores se complementan con una red Mesoescalar capaz de proporcionar información de estaciones meteorológicas con una mayor resolución tanto espacial como temporal. La Red Mesoescalar o Red 
Nacional de Observación Meteorológica de Superficie (RENOMS) en AEMET incluye las redes anteriormente citadas más una serie de Estaciones Meteorológicas Automáticas (EMA) que observan presión, temperatura, viento, humedad, precipitación y tiempo presente $\mathrm{y}$, en el caso de estaciones costeras, temperatura del agua del mar y oleaje, transmitiendo la información cada 10 minutos. La distancia entre estaciones es de 30 a $60 \mathrm{~km}$.

Además AEMET dispone de más estaciones de superficie que junto con las anteriores conforman la Red Climatológica Ordinaria (RCO). En la actualidad la componen aproximadamente 3300 estaciones en funcionamiento que facilitan datos de distintas variables y con distinta periodicidad.

Las estaciones automáticas suministran cada $10 \mathrm{mi}-$ nutos datos de temperatura, precipitación, dirección y velocidad del viento, presión, humedad e insolación. Por otra parte, las estaciones termométricas y pluviométricas, atendidas por personal colaborador, anotan diariamente las temperaturas extremas y la precipitación junto con los meteoros observados y, habitualmente, transmiten sus datos en los primeros días del mes siguiente.

Este elevado número de puntos de observación permiten a AEMET mantener su operatividad diaria en materia de observación, vigilancia y climatología, cumplir con los compromisos nacionales e internacionales en materia de intercambio de datos y facilitar a la sociedad una gran variedad de productos y servicios en tiempo real o casi real a través de su página web y las redes sociales.

Red Climatológica Ordinaria (RCO): A fecha 11 de octubre de 2017 la RCO está constituida por 2902 estaciones, atendidas por personal colaborador; de ellas, 563 son automáticas y las 2339 restantes manuales. Podemos ver su ubicación en el mapa de la Figura 9.6. Las 2339 estaciones manuales registran diariamente la precipitación recogida y los meteoros observados y en aproximadamente 1000 de estas 2339 estaciones se anota también la temperatura máxima y mínima diaria. Las automáticas obtienen al menos datos de temperatura y precipitación, incorporando habitualmente otras variables, principalmente viento y humedad. La frecuencia de observación es diezminutal y la de transmisión cada 4 horas, aunque en algunos casos se transmiten cada hora.

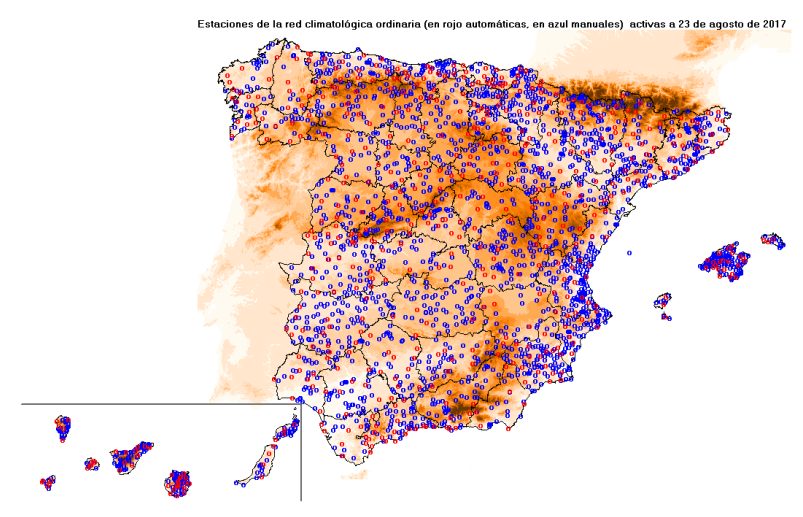

Figura 9.6: Estaciones de la Red Climatológica Ordinaria. Fuente: AEMET.

Su finalidad es constituir una red mínima que permita caracterizar las zonas climáticas del país.

Si nos atenemos a consideraciones climatológicas tales como variables medidas en los distintos tipos de estaciones, presencia o no de personal de AEMET, métodos y periodicidad de transmisión de la información, cabe establecer tres categorías de estaciones:

Estaciones de la red principal: La OMM considera estación principal a las estaciones climatológicas en las que se hacen lecturas horarias, $\mathrm{u}$ observaciones, por lo menos tres veces por día, además de la tabulación horaria de las lecturas de los registradores. En AEMET son las estaciones de la RSBR y parte de las de RSC y están dotadas de personal. Envían sus datos diariamente cuando son grabados y validados en el programa del que disponen, Climatología Sistemas Semi-automáticos (CLISSA) o Climatología Estaciones Completas (CLESCOM). A fecha 11 de octubre de 2017 son 92 estaciones y las podemos ver representadas en el mapa de la Figura 9.7.

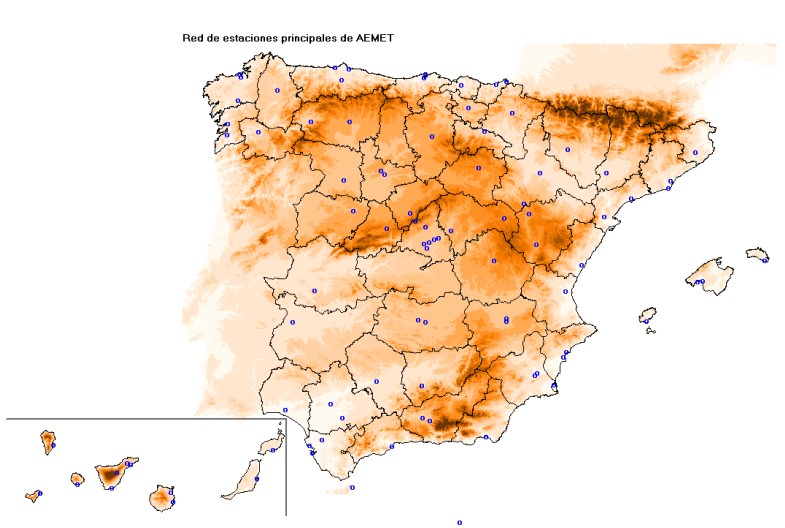

Figura 9.7: Estaciones principales de AEMET. Fuente: AEMET. 


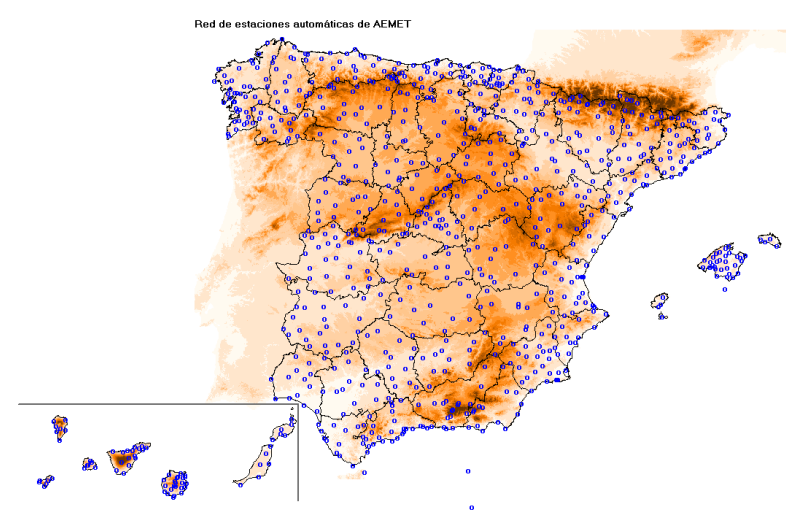

Figura 9.8: Estaciones automáticas de AEMET. Fuente: AEMET.

Estaciones automáticas: Estas estaciones adquieren datos cada 10 minutos que envían al Sistema integrado para la Gestión de las Redes de Observación (SIGROBS) actual concentrador de estaciones automáticas, desde el que son distribuidos a las distintas unidades de AEMET que los demandan, entre ellas el Banco Nacional de Datos Climatológicos. A fecha 11 de octubre de 2017 hay 863 estaciones automáticas en funcionamiento, pertenecientes a la RSC y a la RCO, así como algunas instaladas en estaciones de la RSBR. El mapa de la Figura 9.8 recoge la localización de estas estaciones.

Estaciones termopluviométricas: Son estaciones atendidas por colaboradores, pertenecientes a la RCO. A fecha 11 de octubre de 2017 la componen 2339 estaciones, que anotan diariamente la precipitación diaria y los meteoros observados; de ellas 994 son a la vez termométricas y registran también la temperatura máxima y mínima diaria. Aunque algunas disponen de un software desarrollado por AEMET que les permite mandar los datos en el momento de grabarlos, lo habitual es que los manden en los primeros días del mes siguiente mediante un impreso que una vez escaneado permite extraer los datos mediante OCR (reconocimiento óptico de caracteres). En el mapa de la Figura 9.9 podemos ver la localización de estas estaciones.
El número de estaciones de cada tipo ha ido variando con el tiempo, especialmente las termopluviométricas, tal como recoge el gráfico de la Figura 9.10 en la página siguiente. El número de estaciones pluviométricas aumenta rápidamente desde final de la guerra civil hasta mediados de la década de los 70, pasando de 594 estaciones en 1939 a 5589 en 1976, cayendo notablemente su número posteriormente, lo que nos ha llevado a pasar de 5022 estaciones en 1993 a las 2339 actuales. El número de estaciones termométricas es notablemente menor, ya que la temperatura precisa de un menor número de estaciones para su correcta observación, alcanzándose el máximo en el año 1994 con 2047 estaciones, disminuyendo posteriormente hasta las 994 actuales. El descenso en el número de estaciones termopluviométricas que se observa a partir de los años 90 coincide con la aparición de las estaciones automáticas que, como puede observarse en el gráfico, aumenta muy rápidamente, pasando de 75 en 1996 a 897 en 2014, año en que se alcanza el número máximo, con una ligera disminución posterior hasta las 863 con las que se cuenta a 11 de octubre de 2017.

Estaciones de la red secundaria: La estaciones automáticas y las termopluviométricas conforman la red secundaria de estaciones de AEMET, que a fecha 11 de octubre de 2017 suman 3202 estaciones.

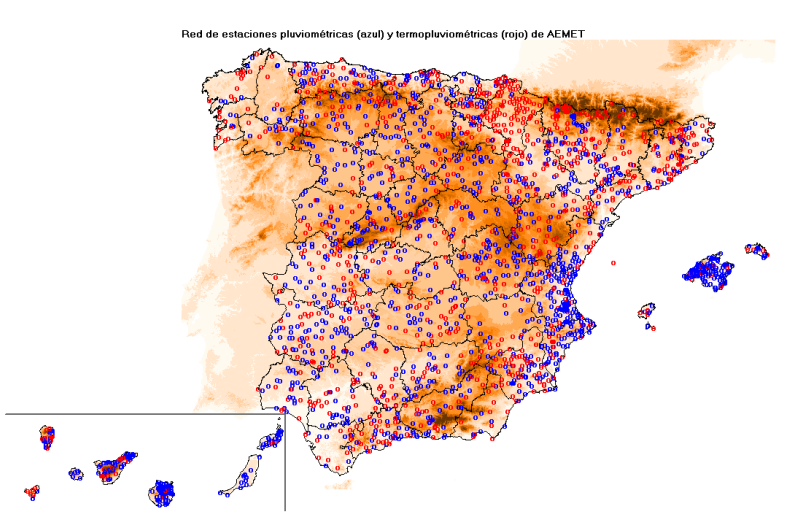

Figura 9.9: Estaciones termopluviométricas de AEMET. Fuente: AEMET. 


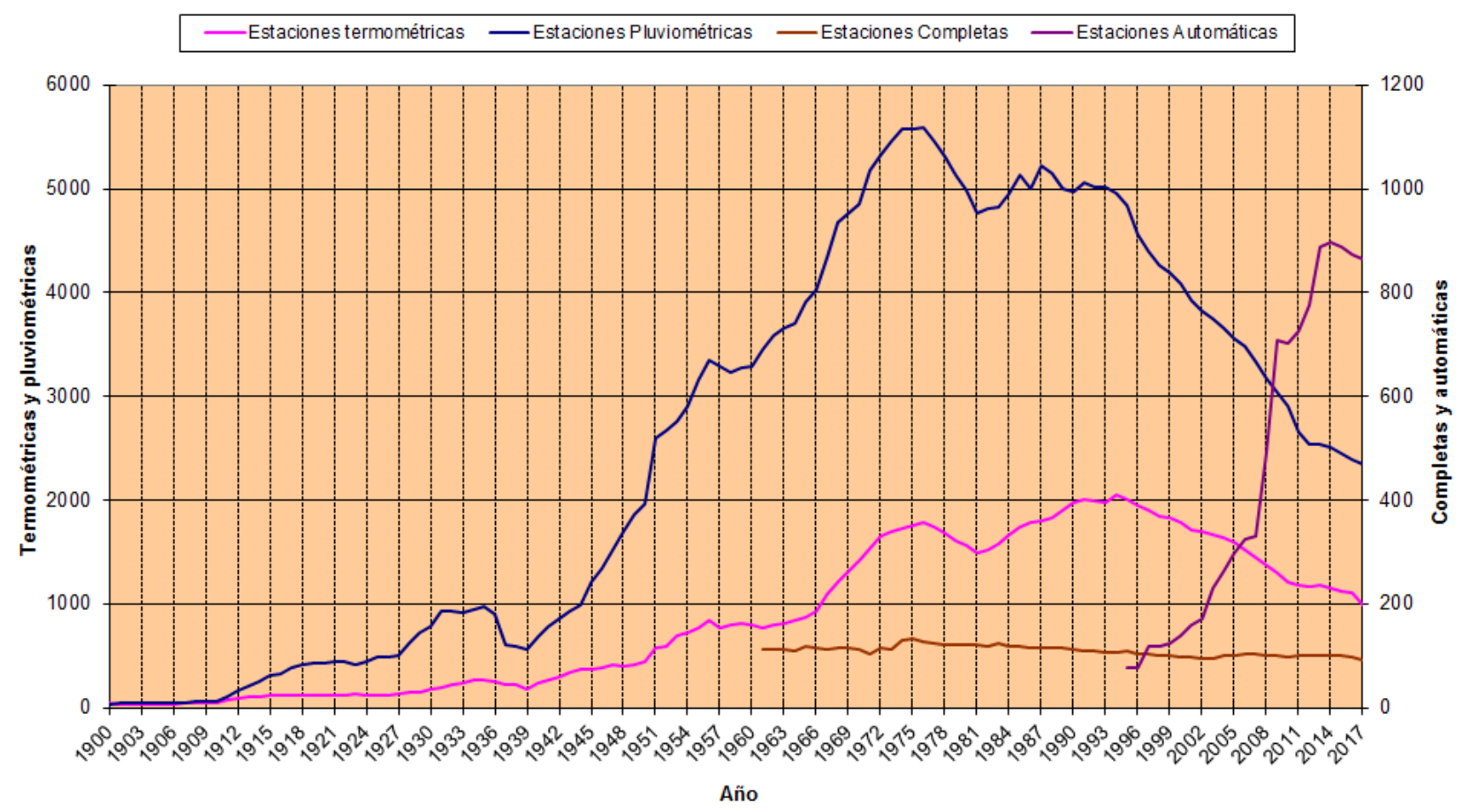

Figura 9.10: Evolución del número de estaciones meteorológicas en AEMET (o INM) desde 1 900. Fuente: AEMET.

\subsection{Almacenamiento de datos y control de calidad}

Los datos procedentes de las distintas estaciones se almacenan en la Base Climatológica, que resulta ser fuente básica de información para el desarrollo de productos y aplicaciones y para la atención de los Servicios Climatológicos ofrecidos por AEMET.

La calidad de la información y de los productos generados depende de la de los datos empleados. Una de las principales preocupaciones del Servicio del Banco Nacional de Datos Climatológicos es asegurar la integridad y calidad de la información que almacena, por eso ha desarrollado un conjunto de procesos de control que, aplicados a los datos antes o después de su incorporación a la Base evitan, en lo posible, la existencia de errores:

- Antes de su almacenamiento en las bases de datos:

- Los datos de estaciones principales y secundarias se controlan a través de los programas de captura que incluyen depuraciones encaminadas a localizar datos que presenten errores de consistencia, continuidad temporal o superen los límites de intervalos de valores posibles, avisan de los errores y permiten su corrección, evitando que se introduzcan en la Base.

- Los datos de las estaciones automáticas no pasan validaciones previas a su carga en la Base para evitar retrasos en su disponibilidad, aunque dentro de las 24 horas posteriores a su carga en la base son tratados por un proceso automático que localiza posibles errores que son validados a diario por personal cualificado.

- Una vez en las bases de datos, los datos se someten a distintos procesos automáticos o manuales para la localización de posibles errores que son estudiados, corregidos o eliminados por el personal técnico de AEMET:

- Control de superación de efemérides con el que se localizan los datos que, cargados durante un día, han superado los valores extremos de la serie a la que pertenezcan.

- Proceso diario automático de depuración de datos diezminutales que localiza errores de los datos de estaciones automáticas almacenados en el día anterior

- Control de coherencia espacial para la localización de datos diarios con coherencia espacial dudosa. Este control se aplica de forma automática a los datos almacenados cada día, pero puede realizarse también de forma ma- 
nual a cualquier periodo de tiempo lo que permite localizar datos históricos que puedan estar afectados de este tipo de error. Existe una versión manual del programa aplicable a datos mensuales.

- Controles ocasionales cuando se sospecha de algún dato como resultado de su aplicación en determinados estudios o tratamientos estadísticos (como por ejemplo estudios de homogeneidad de series). Los errores localizados en estos casos suelen corresponder a datos históricos almacenados con anterioridad a 1995 , no sometidos a los actuales controles de calidad.

Tan importante es la localización de errores como su estudio y verificación por el personal de Climatología. Para ello se dispone de varias herramientas que facilitan esta labor como las gráficas de evolución temporal de distintas variables que pueden mostrar la incoherencia de las observaciones como resultado de un dato incorrecto, imágenes de satélites, radar, mapas de isolíneas, etc. (Figura 9.11).

Actualmente, el Banco Nacional de Datos Climatológicos ofrece información climatológica básica y elaborada a través de la web de AEMET, proporciona a los usuarios información más personalizada y acorde a sus necesidades suministrando los datos necesarios para la elaboración de certificados e informes y atendiendo las solicitudes que por su complejidad o volumen requieren un tratamiento especial, desarrolla nuevos productos, colabora con otras unidades de AEMET en tareas de vigilancia, verificación e información climatológica, y con organismos nacionales e internacionales de manera puntual o continua, proporcionando, por ejemplo:

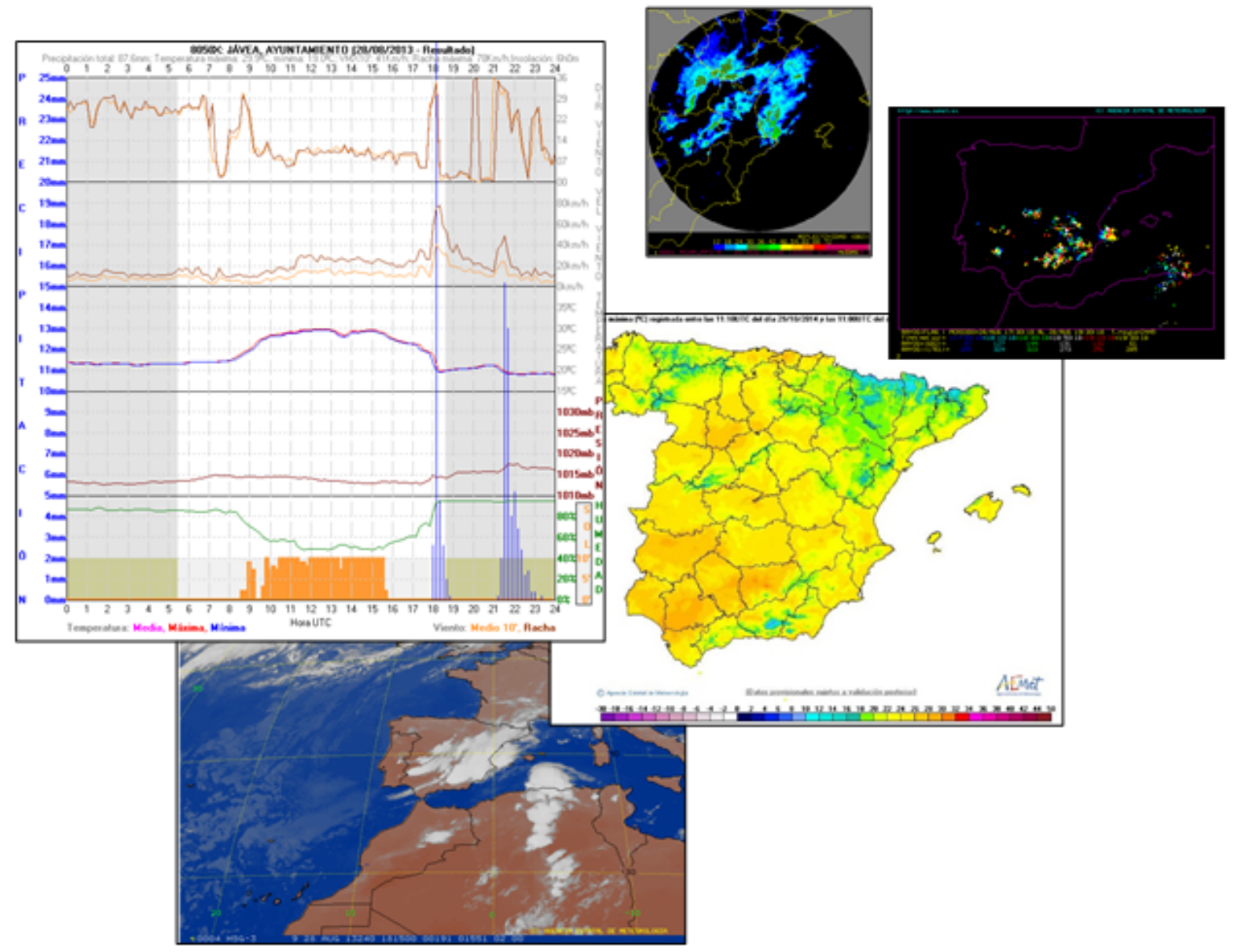

Figura 9.11: Entre las herramientas utilizadas para la detección de errores en las observaciones se encuentran las imágenes de radar y de satélite, los mapas de isolíneas y las gráficas de evolución temporal de distintas variables (fuente: AEMET). 


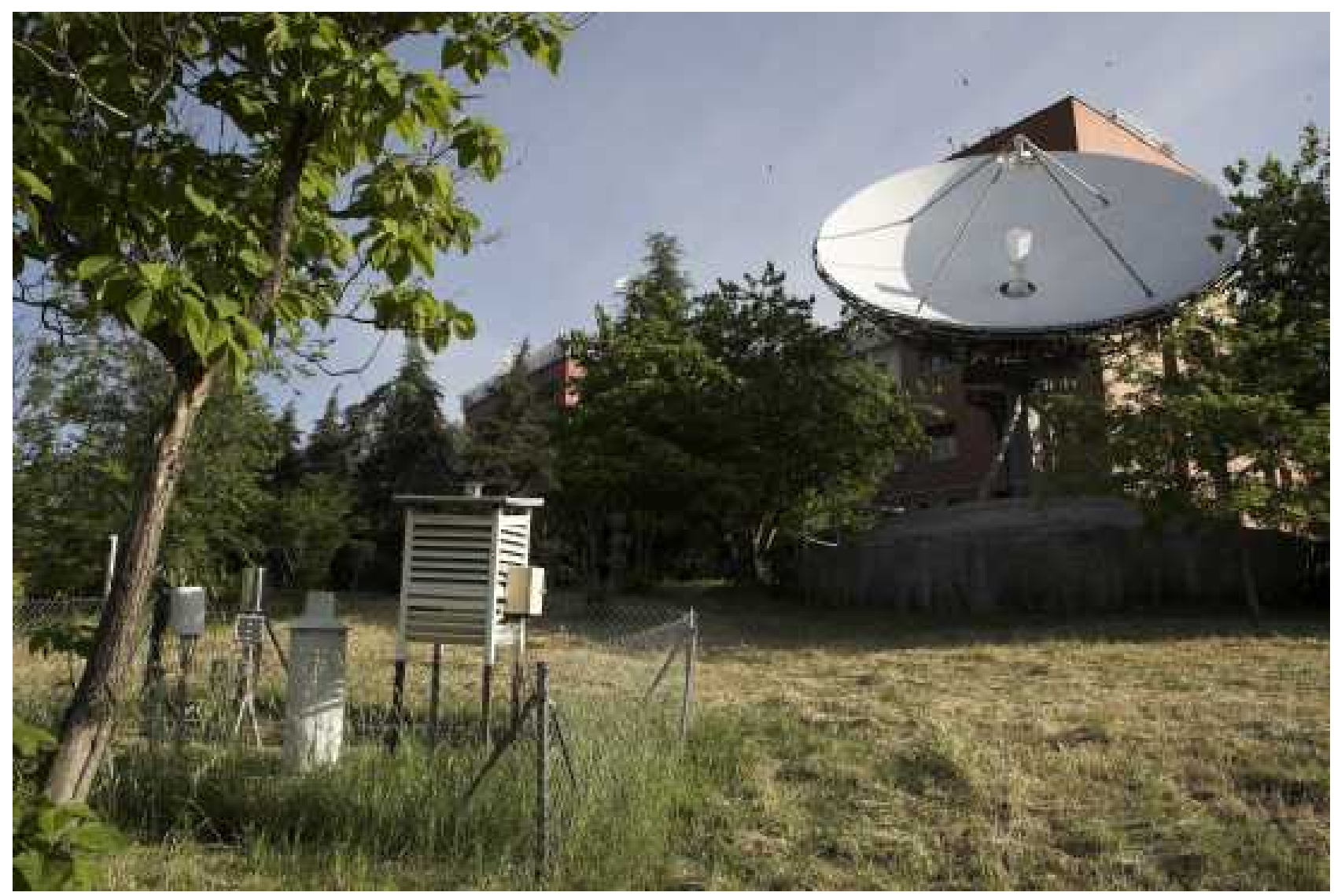

Figura 9.12: Jardín meteorológico de AEMET.

- Datos diarios de 115 estaciones para el proyecto ECA\&D (European Climate Assessment \& Dataset Project) de EUMETNET, cuyo objetivo es el filtrado de las series diarias de observaciones, control de calidad, análisis de extremos, difundiendo tanto los datos de las series (de acuerdo a la política de datos del organismo o estado de origen), como los resultados de los análisis.

- Datos diarios de precipitación de casi 1300 estaciones al GPCC (Global Precipitation Climatology Centre). Este centro está al servicio de los intereses científicos internacionales en lo que se refiere a la vigilancia del ciclo del agua y del clima. Su tarea principal consiste en recopilar y analizar los datos de observaciones convencionales de las precipitaciones, permitiendo la consulta gratuita de productos.

- Datos diarios y mensuales de distintas variables para el grupo WWR (World Weather Records) de la OMM, para su registro y difusión.
- Información fenológica al Pan European Phenology Project.

- Colaboraciones para la determinación del Estado Mundial del Clima de la OMM y publicaciones relacionadas.

A pesar de la continua vigilancia realizada sobre los datos almacenados en la Base Climatológica, la fuerte demanda de información, sobre todo en tiempo real, impide realizar controles suficientes que eviten la difusión de errores en algunas ocasiones. Es primordial evitarlo y por eso se sigue trabajando en las tareas de control, mejorándolas de forma continua, añadiendo nuevas pruebas y sometiendo a los datos a tantas depuraciones como se crea necesario. Como consecuencia de ésto, la Base Climatológica puede considerarse un sistema de almacenamiento vivo, cuya información puede variar en cualquier instante como resultado de estos chequeos continuos y siempre con el resultado de una mejora de la calidad del dato. 


\subsection{Referencias}

[1] AEMET/IM. Atlas climático ibérico. Temperatura del aire y precipitación (19712000). Agencia Estatal de Meteorología. Ministerio de Medio Ambiente y Medio Rural y Marino, 2011 (citado en página 118).

[2] PACHAuri, Rajendra K y col. Climate change 2014: synthesis report. Contribution of Working Groups I, II and III to the fifth assessment report of the Intergovernmental Panel on Climate Change. IPCC, 2014 (citado en página 120).

[3] Sneyers, Raymond y col. On the statistical analysis of series of observations. 143. World Meteorological Organization (WMO),
1991, página 192. ISBN: 9263104158 (citado en página 119).

[4] Trewin, Blair C. "The role of climatological normals in a changing climate". En: WMO-TD 1377 (2007) (citado en página 120).

[5] WMO. Guide to climatological practices. third. WMO. World Meteorological Organization, 2011. ISBN: 9789263101006. URL: http : / / library . wmo. int/pmb_ged/ wmo_100_en . pdf (citado en páginas 118, 119).

[6] WMO. Global Observing System Components, WMO. 2017. URL: https : / / www . wmo. int/pages/prog//www/OSY/Gos components . html (visitado 16-11-2017) (citado en página 122). 